\title{
EFFECT OF HEAVY ION IRRADIATION ON THE MAGNETIC PROPERTIES OF $\mathrm{CuGeO}_{3}$
}

L. GladczuK ${ }^{a}$, E. Mosiniewicz-Szablewska ${ }^{a}$, R. SzYMCZAK $^{a}, \mathrm{M}$ BaraN $^{a}$, H.A. DAzBKowska ${ }^{b}$, J. ChOIŃSKI ${ }^{c}$, T. CZOSNYKA ${ }^{c}$ AND H. SzYMCZAK ${ }^{a}$

${ }^{a}$ Institute of Physics, Polish Academy of Sciences

Al. Lotników 32/46, 02-668 Warsaw, Poland

${ }^{b}$ Brockhouse Institute for Materials Research, McMaster University, Hamilton, Canada

${ }^{c}$ Heavy Ion Laboratory, Warsaw University, Warsaw, Poland

The influence of heavy ion irradiation on the magnetic properties of the spin-Peierls compound $\mathrm{CuGeO}_{3}$ has been investigated by means of the electron paramagnetic resonance, the spontaneous magnetization, and the low field ac susceptibility. The measurements were performed on the $\mathrm{CuGeO}_{3}$ single crystals before and after irradiation with $80 \mathrm{MeV}$ oxygen ions in the wide temperature range of $2-300 \mathrm{~K}$. It was found that the irradiation induced an increase in the EPR signal intensity, magnetization, and susceptibility of this material. It was also shown that the irradiation does not lead to a decrease in the spin-Peierls transition temperature and to the formation of an antiferromagnetic phase which is expected to appear in disordered spin-Peierls systems.

PACS numbers: $75.30 . \mathrm{Kz}, 75.50 . \mathrm{Ee}, 76.30 . \mathrm{Da}$

\section{Introduction}

The behavior of spin-Peierls (SP) systems in the presence of disorder has recently attracted much attention. The compound $\mathrm{CuGeO}_{3}$ is one of these systems and undergoes the SP transition at $T_{\mathrm{SP}}=14 \mathrm{~K}[1]$. The orthorhombic structure of this material is composed of linear $\mathrm{CuO}_{2}$ chains running along the $c$ axis, well separated from each other by $\mathrm{GeO}_{2}$ chains [2]. Below the $\mathrm{SP}$ transition temperature the $\mathrm{GeO}_{2}$ chains dimerize and a gap in the spin excitation spectrum opens [3].

Recently, it has been demonstrated that the SP transition in $\mathrm{CuGeO}_{3}$ is drastically affected by doping which leads to a linear reduction of $T_{\mathrm{SP}}$ and to the appearance of an antiferromagnetically ordered phase at $T<5 \mathrm{~K}$ [4]. The coexistence of antiferromagnetism and dimerization is suggested to be induced by the disorder resulting from the chemical substitutions [5].

In order to obtain a deeper insight into the effect of disordering on the SP state, we present a detailed study of magnetic properties of the heavy ion irradiated $\mathrm{CuGeO}_{3}$ single crystals. 


\section{Experimental}

A single crystal of $\mathrm{CuGeO}_{3}$, used in the experiment, was grown from a $\mathrm{CuO}$ flux using a method described in Ref. [6]. The same sample was subsequently three times irradiated with $80 \mathrm{MeV}$ oxygen ions in the same experimental geometry at room temperature. In each case the irradiation dose was $10^{14} \mathrm{ions} / \mathrm{cm}^{2}$. The beam of $\mathrm{O}$ ions was perpendicular to the largest crystal surface, plane $(b c)$. Using the TRIM [7] simulation we estimated that in our case only $15 \%$ of the sample volume was subject to irradiation damage. The magnetization measurements were carried out using a superconducting quantum interference device (SQUID) magnetometer (MPMS-5) in magnetic fields up to $5 \mathrm{~T}$. EPR spectra were recorded using a standard X-band spectrometer (Bruker $300 \mathrm{E}$ ) operating at $9.5 \mathrm{GHz}$ with $100-\mathrm{kHz}$ field modulation. The measurements were performed for an external magnetic field applied along each of the three crystallographic directions in the wide temperature range of $2-300 \mathrm{~K}$.

\section{Results and discussion}

The influence of heavy ion irradiation on the temperature dependence of the magnetic susceptibility is demonstrated in Fig. 1. After irradiation a large increase in the susceptibility is observed at low temperatures which depends on the irradiation dose. Above $T_{\mathrm{SP}}=14 \mathrm{~K}$ the difference between the susceptibilities before and after irradiation decreases and for $T>150 \mathrm{~K}$ is within the error limits. The intensity of the EPR signal follows a similar behavior (see the inset of Fig. 1). It is also found that the irradiation does not lead to a decrease in the SP transition temperature and to the reduction of $g$-factor as it is expected in doped $\mathrm{CuGeO}_{3}$

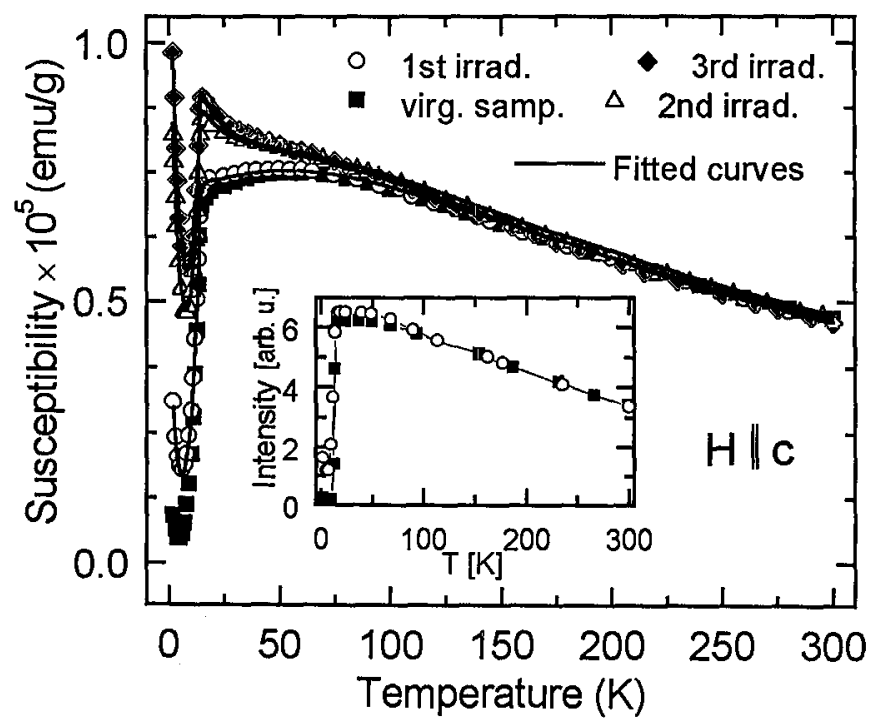

Fig. 1. Temperature dependences of the magnetic susceptibility and the EPR signal intensity for $\mathrm{CuGeO}_{3}$ before and after heavy ion irradiation. 
crystals. It does not also produce appreciable changes in the line width of the EPR signal. The susceptibility of the irradiated sample can be expressed as

$$
\chi_{\mathrm{irr}}(T)=(1-D) \chi_{\mathrm{virg}}(T)+D\left(\chi_{\mathrm{CW}}+\chi_{\mathrm{BF}}\right),
$$

where the first term describes the susceptibility of nondefected chains which are present in the unirradiated part of the sample and may also exist in the irradiated layer and the second term describes the effective susceptibility of the destroyed chains. $D$ is the ratio between the volume of the irradiated part and the total volume of the sample, $\chi_{\mathrm{CW}}$ is the Curie-Weiss component, and $\chi_{\mathrm{BF}}$ is the Bonner-Fisher component describing the susceptibility of the broken $\mathrm{Cu}-\mathrm{O}$ chains.

$\chi_{\mathrm{CW}}$ and $\chi_{\mathrm{BF}}$ are described as [8]

$$
\chi_{\mathrm{CW}}=C /(T-\theta), \quad \chi_{\mathrm{BF}}=B F(x) / T,
$$

where $C$ and $B$ are proportional to the numbers of isolated $\mathrm{Cu}^{2+}$ ions and $\mathrm{Cu}^{2+}$ ions in broken $\mathrm{Cu}-\mathrm{O}$ chains, respectively. $F(x)$ is the quadratic/cubic function of the argument $x=|J| / k_{\mathrm{B}} T$, with $J$ being the $\mathrm{Cu}^{2+}-\mathrm{Cu}^{2+}$ intrachain superexchange coupling constant [8].

Using the procedure described above to fitting the temperature dependences of susceptibility for irradiated samples, we have found that the increase in the volume of the effectively irradiated part of the sample is practically linear with irradiation dose and reaches, after the third irradiation, a value corresponding to the calculated penetration depth. It was also noticed that both the number of isolated $\mathrm{Cu}^{2+}$ ions and the number of $\mathrm{Cu}^{2+}$ ions in broken $\mathrm{Cu}-\mathrm{O}$ chains increase with increasing irradiation dose due to the decreasing number of $\mathrm{Cu}^{2+}$ ions in dimerized chains.

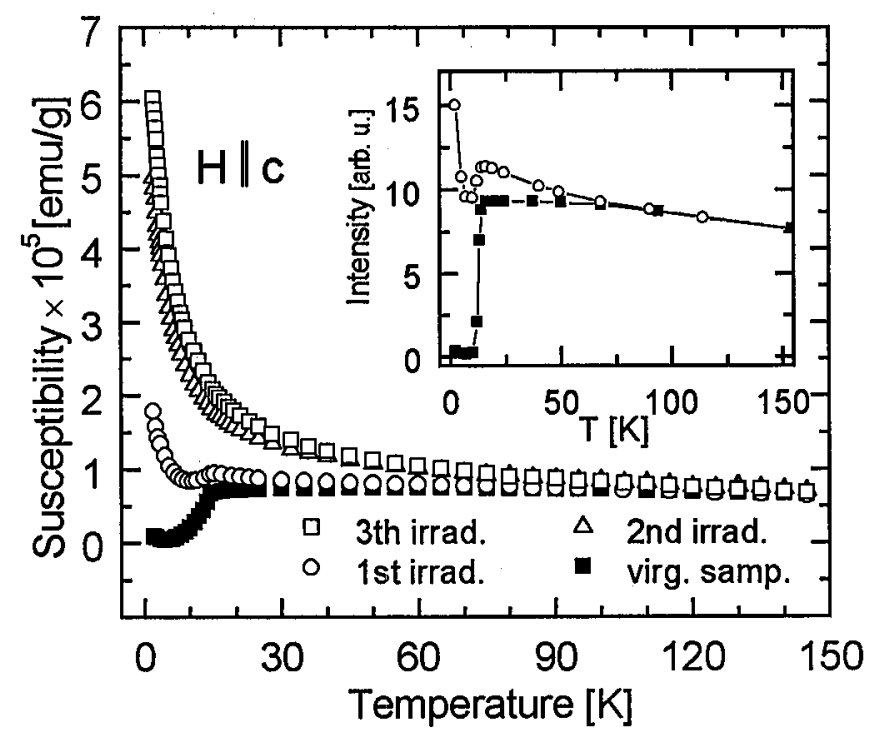

Fig. 2. Temperature dependences of the magnetic susceptibility and the EPR signal intensity for $\mathrm{CuGeO}_{3}$ after subtracting the susceptibility for the unirradiated part of the sample. 
Figure 2 presents the susceptibilities of irradiated samples after the susceptibility of the unradiated part of the sample is subtracted. The results clearly show a large increase in the Curie-Weiss component and a complete suppression of the SP transition for higher irradiation doses. Similar effects were obtained using the same procedure for the EPR signal intensity (see the inset of Fig. 2).

\section{Conclusions}

Our results show that the heavy ion irradiation does not lead to a decrease in the SP transition temperature which is in contrast to the case of chemical doping. Furthermore, we do not observe the formation of an antiferromagnetic phase which is expected to appear in disordered spin-Peierls systems. It could be explained by assuming that $\mathrm{Cu}-\mathrm{O}$ chains are effectively less influenced by the irradiation defects than by chemical substitution, which causes chemical pressure in the lattice. It seems that disorder connected with cutting of the chains destroys the one-dimensional ordering but it does not have to lead to the formation of a three-dimensional magnetic ordered state at low temperatures.

\section{Acknowledgments}

This work was supported in part by the Committee for Scientific Research under contract No. 2 P03B 13615.

\section{References}

[1] M. Hase, I. Terasaki, K. Uchinokura, Phys. Rev. Lett. 70, 3651 (1993).

[2] H. Völlenkle, A. Wittmann, H. Nowotny, Monatsch. Chem. 98, 1352 (1967).

[3] M. Nishi, O. Fujita, J. Akimitsu, Phys. Rev. Lett. 50, 6508 (1994).

[4] L.P. Regnault, J.P. Renard, G. Dhalenne, A. Revcolevschi, Europhys. Lett. 32, 579 (1995).

[5] M. Fabrizo, R. Mélin, Phys. Rev. B 56, 5996 (1997).

[6] M.D. Lumdsen, B.D. Gaulin, H. Dąbkowska, Phys. Rev. B 57, 14097 (1998).

[7] J.P. Biersack, L.G. Haggmark, Nucl. Instrum. Meth. 174, 257 (1980).

[8] W.E. Hatfield, J. Appl. Phys. 52, 1985 (1981). 